\title{
Maternal mortality in Sierra Leone: from civil war to Ebola and the Sustainable Development Goals
}

\author{
Carah Alyssa Figueroa ${ }^{1}$ - Christine Lois Linhart ${ }^{1}$ - Walton Beckley ${ }^{2} \cdot$ \\ Jerico Franciscus Pardosi ${ }^{1,3}$
}

Received: 26 September 2017/ Accepted: 21 November 2017/Published online: 27 November 2017

(c) Swiss School of Public Health (SSPH+) 2017

More than 1 year has passed since Sierra Leone marked the end of the largest Ebola virus epidemic ever recorded. However, maternal mortality remains a major challenge. The country's maternal mortality ratio (MMR) is the highest in the world, estimated by the UN Maternal Mortality Estimation Inter-Agency Group to be 1360 [80\% uncertainty interval (UI), 999-1980] deaths per 100,000 live births (WHO et al. 2015). This is higher than what the Demographic and Health Survey reports (1165 per 100,000 live births), given incomplete civil registration and vital statistics (CRVS) (Statistics Sierra Leone and ICF International 2014). In contrast, the Millennium Development Goal (MDG) 5A target the country needed to achieve by 2015 was 450 maternal deaths per 100,000 live births.

The inadequate reduction in MMR must be assessed within the country's challenging context. In 1990, the MMR was estimated to be 2630 (80\% UI 1780-3640) per 100,000 live births. The civil war (1991-2002) collapsed the economy and health infrastructure, resulting in unattended birth deliveries and unavailable emergency obstetric care (EmOC). This culminated in an increase in deaths of mothers and their newborns (Milland and Bolkan 2015). Accordingly, between 1990 and 2000, the MMR declined

Jerico Franciscus Pardosi

jerico.pardosi@unsw.edu.au

1 School of Public Health and Community Medicine, Faculty of Medicine, University of New South Wales, Sydney, Australia

2 Pharmaceutical Society of Sierra Leone, Freetown, Sierra Leone

3 National Institute of Health Research and Development, Ministry of Health, Jakarta, Indonesia by $-0.1 \%(80 \%$ UI -2.8 to $2.2 \%)$ per year (WHO et al. 2015).

Between 2000 and 2015, the MMR reduced by $4.4 \%$ (80\% UI 1.6-6.2\%) per year (WHO et al. 2015). During the post-conflict period, there were improvements across the health sector (Ministry of Finance and Economic Development 2016). More deliveries occurred in health facilities (52\% in public health facilities, $2 \%$ in private), more pregnant women attended at least four antenatal care visits (76\%) and more births were assisted by skilled health personnel $(60 \%)$ (Statistics Sierra Leone and International 2014). The Government introduced the Free Health Care Initiative in 2010 to provide essential healthcare services to expectant and new mothers. However, upsurges in demand led to frequent resource shortages, out-of-pocket spending, and overburdened health personnel (Maxmen 2013). Only 1.2 properly functioning EmOC facilities per 500,000 population exist, and $2 \%$ of births occur in an EmOC facility ( $10 \%$ at any facility type) - short of the recommendations for at least five EmOC facilities per 500,000 population, and $15 \%$ of expected births at EmOC facilities (Jones and Ameh 2015). The capacity of health systems to meet maternal healthcare needs was severely limited in Sierra Leone.

The Ebola epidemic from May 2014 to November 2015 is presumed to have further deteriorated the health system, setting back the progress made towards achieving MDG5A. Considering the significant loss of health workers, one study estimates the MMR increased to levels seen in the year 2000 (Evans et al. 2015). The closure of major health facilities exacerbated existing inequities in healthcare access. Facility-based deliveries and antenatal care visits reduced by about $25 \%$, driven by mistrust of health workers and preferences for traditional birth attendants. Health staff were also reluctant to care for pregnant women 
due to the lack of infection control measures (Jones and Ameh 2015). Consequently, the "three delays" which increase maternal mortality risk were exacerbated during the Ebola epidemic: delays in deciding to seek care, reaching a health facility, and receiving appropriate and quality care (Thaddeus and Maine 1994).

Sierra Leone faces immense challenges in the Sustainable Development Goals (SDGs) era. The SDG3A target is to reduce the global MMR to less than 70 per 100,000 live births by 2030 (Ministry of Finance and Economic Development 2016). The national strategic plan, the Agenda for Prosperity (A4P) 2013-2018, reflects a commitment to achieving SDG3A (Government of Sierra Leone 2013). Although this is laudable, one must consider the increased burden on resources and management to periodically report progress on achieving SDG3A-as well as other targets. Recognising these concerns, the government aims to establish international benchmark systems that increase efficiency and minimise misreporting (Ministry of Finance and Economic Development 2016). In this regard, it is important to have indicators that reflect the specific needs and realities of Sierra Leoneans. More importantly, to effectively monitor progress towards SDG3A, investments are needed to build the capacity of integrated health information systems, particularly establishing a robust infrastructure for CRVS and training professionals in data collection, management and reporting. This should form part of other health system strengthening commitments addressed in the A4P, including upgrading health facilities and expanding the health workforce (Government of Sierra Leone 2013).

Beyond the mainstream health sector, the A4P also focuses on multi-sectoral measures and addressing social inequities, which are integral to facilitating equitable reductions in maternal mortality (Government of Sierra Leone 2013). Addressing mistrust and sociocultural barriers to using maternal health services would further contribute to improving equity. Indeed, since the MDG-SDG transition, increasing attention has been paid to the importance of equity in maternal health care utilization for reaching the SDG targets (Çalışkan et al. 2015).

Sierra Leone bears the world's highest MMR considering the challenges of fragmented health systems in the context of civil war and the Ebola epidemic in the MDGs era. To drive progress towards SDG3A, national plans to strengthen entire health systems, particularly health information systems, and improve equity in healthcare access must be translated into action, coupled with sustained efforts to restore trust between local communities and the health system-and crucially, supported by strong political commitment.

\section{Compliance with ethical standards}

Conflict of interest The authors declare no conflict of interest.

\section{References}

Çalışkan Z, Kılıç D, Öztürk S, Atılgan E (2015) Equity in maternal health care service utilization: a systematic review for developing countries. Int J Public Health 60:815-825

Evans DK, Goldstein M, Popova A (2015) Health-care worker mortality and the legacy of the Ebola epidemic. Lancet Glob Health 3:e439-e440. https://doi.org/10.1016/s2214-109x(15) 00065-0

Government of Sierra Leone (2013) Sierra Leone's third generation poverty reduction strategy paper (2013-2018) — the agenda for prosperity—road to middle-income status. http://www.undp.org/ content/dam/sierraleone/docs/projectdocuments/povreduction/ undp_sle_The $\% 20$ Agenda\%20for $\% 20$ Prosperity $\% 20$.pdf. Accessed 1 Aug 2017

Jones S, Ameh C (2015) Exploring the impact of the Ebola outbreak on routine maternal health services in Sierra Leone VSO, Liverpool, UK. https://www.vsointernational.org/sites/default/ files/VSO\%20Sierroa\%20Leone\%20-\%20Impact\%20of\%20Ebola. pdf. Accessed 4 June 2017

Maxmen A (2013) Sierra Leone's free health-care initiative: work in progress. Lancet 381:191-192. https://doi.org/10.1016/S01406736(13)60074-4

Milland M, Bolkan HA (2015) Enhancing access to emergency obstetric care through surgical task shifting in Sierra Leone: confrontation with Ebola during recovery from civil war. Acta Obstet Gynecol Scand 94:5-7. https://doi.org/10.1111/aogs. 12540

Ministry of Finance and Economic Development (2016) United Nations Sustainable Development Goals - the 2030 agenda for sustainable development-advanced draft report on adaptation of the goals in Sierra Leone. Government of Sierra Leone

Statistics Sierra Leone, ICF International (2014) Sierra Leone Demographic and Health Survey 2013. SSL and ICF International, Freetown

Thaddeus S, Maine D (1994) Too far to walk: maternal mortality in context. Soc Sci Med 38:1091-1110

WHO, UNICEF, UNFPA, World Bank Group, United Nations Population Division, Maternal Mortality Estimation Inter Agency Group (2015) Maternal mortality in 1990-2015-Sierra Leone 